\section{Networking Solution For PDP-11 Based EDS And Image Acquisition Systems} John Mansfield, University of Michigan

One of the major difficulties with the older DEC PDP-11 based EDS systems is that they were designed as standalone units. Their manufacturers believed that the systems were capable of performing all the spectral processing that the users would ever need, and therefore did not allow for transfer of data to other computer systems. While some of the vendors have attempted to offer file transfer capabilities as upgrades, typically these upgrades are based on a serial connection using a file transfer program like Kermit. Although such solutions are acceptable for the transfer of small numbers of spectra, they cannot seriously handle hundreds of spectra or digital images data without tying up the EDS computer for extended periods of time. In multi-user environments, it is often desirable to move the data taken in one users microscope session immediately, to avoid filling to capacity the disk space on the acquisition system and rendering the system unusable for the next user.

The best solution for this problem is to install an ethernet networking card in the PDP-11 system and use the much higher transfer rates offered by this form of communication as compared to a serial connection. As an example of what is needed to effectively network a PDP-11 based system, I will describe the Tracor/Noran TN5502 system that we have successfully networked at the University of Michigan. We have also connected three Kevex systems to the net and I have colleagues who have used this method with EDAX systems also.

Networking an EDS computer requires that there is a free slot in the backplane of PDP-11. Into this slot one must install a DEQNA ethernet card. I am uncertain whether DEC still offer these cards, but there are a variety of third party vendors where you can buy a reconditioned card. The last card I purchased was $\sim \$ 600$. It usually necessary to purchase a secondary adapter to connect the card to the local ethernet, depending on the "flavor" of ethernet that exists in the building in which the installation is taking place

There are several different software packages that may be used to communicate from the PDP-11 to other computers on the net. For our systems we have used TCP/IP software marketed by a company called Process Software (address as follows). TCP/IP is an acronym which stands for Transmission Control Protocol/nternet Protocol. TCP/IP is a industry standard protocol for transferring data from computer to computer, whether this data is in the form of files, email or a remote terminal session. The "Internet" that you may have heard mentioned frequently uses largely TCP/IP for communication. The file transfer software used to move files over the network is called ftp (file transfer protocol). The Process Software ftp software currently lists for around $\$ 1200$, multiple copies at one location receive a discount. With the Process Software package one can initiate connections from the PDP and connect to any other computer that is running ftp (client) or one can leave the ftp software running on the PDP and connect to it from a remote machine (server). In the case of the TN5502, since it does not use the standard DEC operating system, it is necessary to buy a license for DEC's RT-11 (the single user operating system used by Kevex, EDAX and others in their PDP based systems) in order to run the ftp program. A license for RT- 11 currently lists at $\$ 960, \$ 1600$ with media. There are a variety of discounts available for government labs and educational institutions

In order to run the ftp software and use the EDS system in the normal manner, both the Tracor system software and the RT-11 software have to be available on the PDP-11 simultaneously. I have a hard disk installed on my TN5502 together with a Syquest cartridge drive. Under the Tracor/Noran system I then have 4 hard disks, drives 1 through 4 . I load the Noran software on drive 1 and the RT- 11 operating system onto drive 2 and turn the system autoboot off. Thus, when the system is turned on or reset, the printer will print "START?" To use the native TN system one simply responds " $Y$ " or enters "DL0" for RT-11 ano network access one types "DL1". When RT-11 boots there are still four drives but they are now labeled DLO, DL1, DL2 and DL3. The fact that one may type

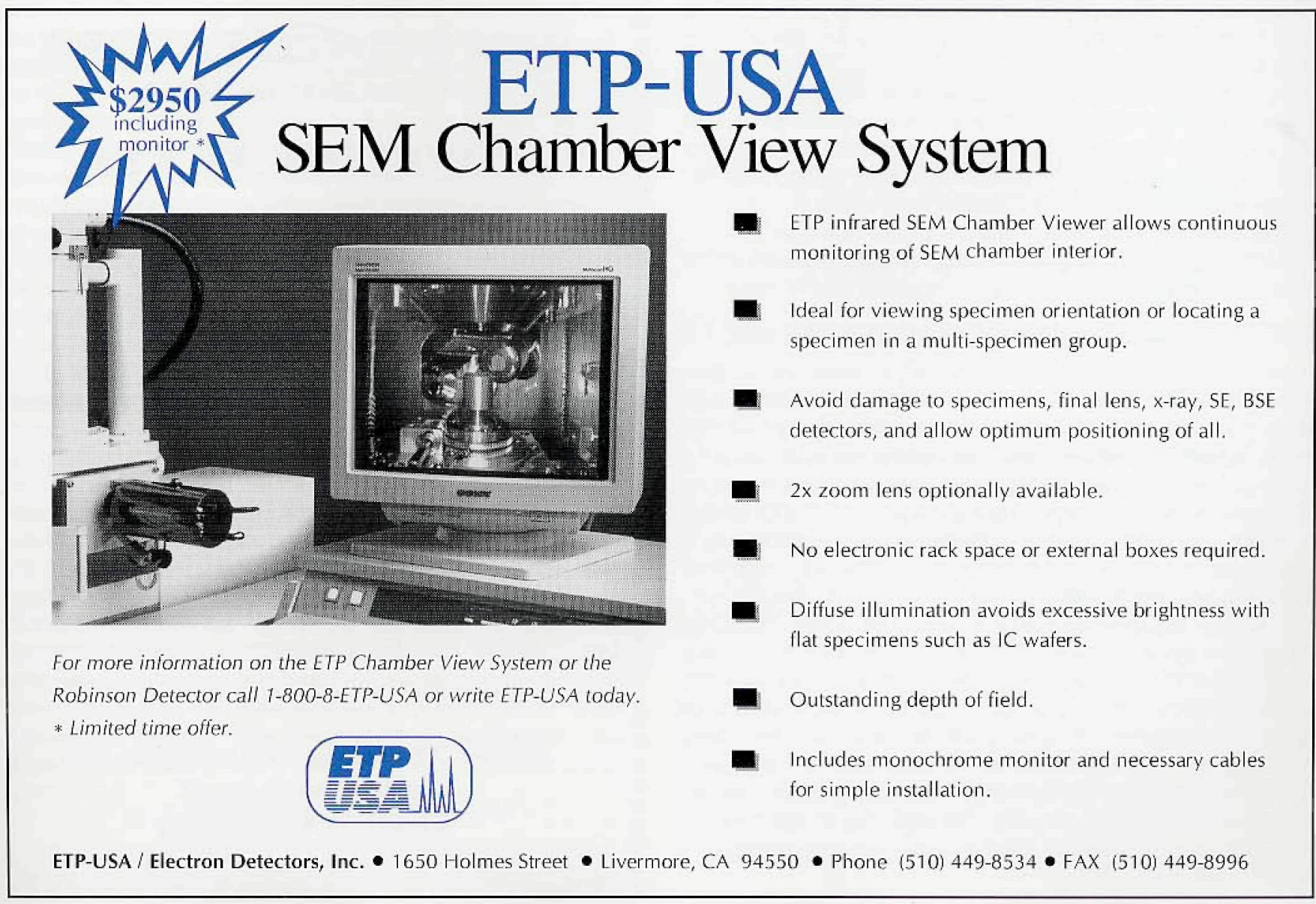


the names of these drives to boot the system, either for the Tracor operating system or RT-11, indicates that the two systems share a common root.

Once one has collected data and wishes to transfer it the system must be rebooted into RT-11. This is inconvenient, but the only way to obtain network access, since no TCP/IP drivers were written for the Tracor operating system. The RT-11 system can be configured to automatically load the ftp software and set the default disk to be the data disk. After file transfer the system must be rebooted again to use the EDS capabilities.

The Process Software ftp program is hardware keyed. One must run an ID program on the EDS PDP-11, then call Process Software and report the numbers displayed by the ID program. The company then allocate a series of numbers that act as a key so that one may run the software on that particular PDP. 11. This may sound tedious, but once one has entered the key in a specially named text file, it never needs to be entered again. I have used this program for nearly 5 years and it has performed flawlessly in that time. The Process Software ftp program is a little primitive when compared with ftp programs for UNIX hosts, PC or Macs. If run as a client, i.e. the transfer is initiated from the PDP11 , it does not support wild cards and thus the name of each file to be transferred must be entered. If run in server mode, however, one can issue batch commands from the remote to program and transfer large numbers of files with a single command. This is the mode that I always use. A schematic of the networked system is illustrated in figure 1.

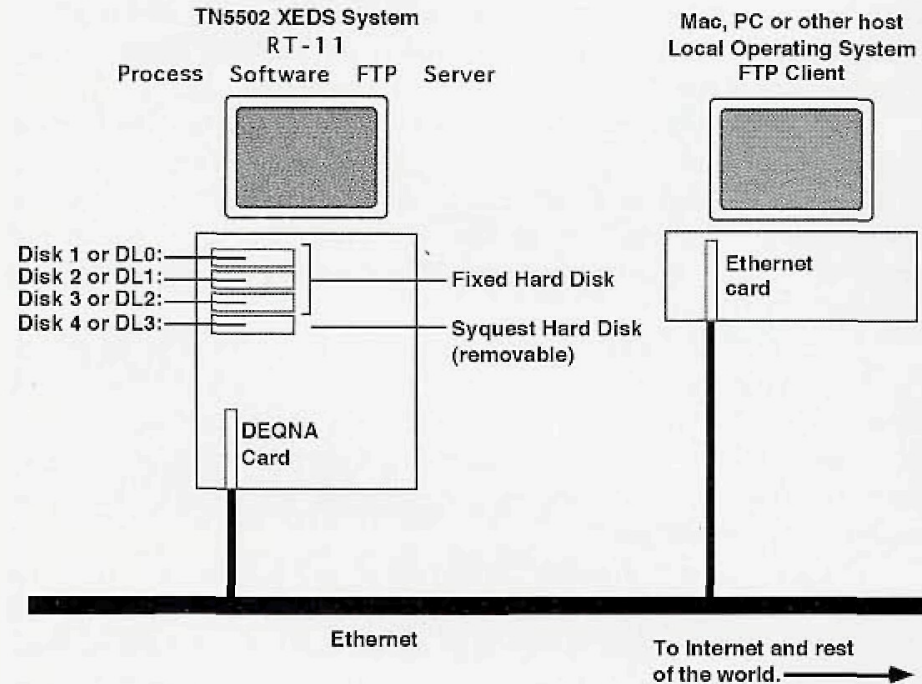

Although the files can be transferred anywhere on the Internet, typically we transfer data to either Macs or PCs. For transfer to Macs we use the freebie ftp client from Dartmouth College "Fetch". The current version is 2.1.2 and it is both $680 \times 0$ and PowerPC compatible (i.e. the program is a fat binary and contains native code for both types of Mac). For transfer to the PC we use LAN Workplace of DOS, which integrates with our NetWare software. Most file transfer is done to the Macs. Spectral data may be directly read into the NIST DeskTop Spectrum Analyzer (DTSA) program ( $\$ 790$ available directly from NIST in Maryland or from 4Pi Analysis in North Carolina). Images may be read into a variety of image processing programs, NIH-Image, Photoshop, etc., after conversion with a free utility called TiffMaker, witten by Hal Estry who is in our laboratory at the University of Michigan.

If you require further information on networking PDP-11 based data acquisition systems I may be able to answer your questions. The best way to contact me is via email, with relevant information below. Opinions expressed in this document are my own and do not necessarily reflect those of my employers.

John Mansfield

eMail: John.F.Mansfield@umich.edu

Tel.: (313)936-3352 Fax: (313)936-3352

* Process Software is at:

959 Concord Street, Frarmingham, MA 01701

Their phone number is: $800-722-7770$

\section{Exploiting the Binocular Head in Polarized Light Microscopy}

Walter C. McCrone, McCrone Research Institute

Having been brought up on monocular microscopes 1 find the omnipresent binocular systems a luxury. To support this viewpoint I'd like to suggest some benefits you may not have considered.

Because l'm used to monocular viewing I sometimes use two different oculars, say $10 \mathrm{X}$ and $25 \mathrm{X}$ in order to scan quickly to find an area of interest and then to examine the detail with higher magnification. Occasionally I use both oculars simultaneously and "concentrate" on either image to the exclusion of the other. A better way is to set the interocular distance at the extreme setting most different from your own interocular distance. By moving your head about a centimeter either way you can use either ocular.

A variation of this theme is very helpful when you are trying to find good crystals showing useful interference figures. I simply replace one ocular with a pinhole (thoughtfully supplied by the manufacturers with all polarized light microscopes - for reasons of habit only, I'm sure). The pinhole yields a small but very sharp interference figure without the Bertrand lens. With the ocular tubes set for normal binocular viewing one can observe each crystal and, superimposed on it, the corresponding interference figure. One can, by this means, scan a field of dozens of crystals in a minute or so to select a useful view. Only the crystal or area at the very field-center will contribute to the interference figure. For measurements of optic axial angle one then inserts the Bertand lens for normal conoscopic viewing of the crystal located by the pinhole method.

Finally, stereo imaging is easy by changing the interocular distance a few $\mathrm{mm}$ so that each eye looks through an outer (properly stereoscopic) or inter (pseudo-stereoscopic) edge of the oculars. I can think of some other ideas but they seem a bit too far out even for me (e.g., having an analyzer over one ocular would give crossed polars with one eye to compare with the single polar view; a very tiny, about one $\mathrm{mm}$, aperture at the eyepoint of one ocular would yield annular stop dispersion staining; a tiny one $\mathrm{mm}$ opaque stop similarly placed would yield central stop dispersion staining, etc., etc.). Still, one other practical idea is to insert a light meter in one ocular tube to record light intensity of fusion preps as they are heated to detect solid-solid phase changes and melting points you observe directly through the other ocular.

\section{More Help???}

Regarding this newsletter, we are continuing in our efforts to both increase and decrease the size of our mailing list.

As to increasing readership, and should you start to feel the publication of interest and value, we would appreciate it if you would route a copy to others in your organization with an interest in microscopy. All we need is name and proper address to start a no cost subscription. This request applies to both U.S. and "overseas" readers.

And we must remove those from our mailing list who either do not wish to receive a copy or are not receiving their copy due to a wrong address. A "?" following your name on the mailing address indicates that we are not certain if you wish to receive, or are receiving, a copy. Should you be in this category, and wish to continue to receive the newsletter, kindly complete the enclosed questionnaire. 